University of Nebraska - Lincoln

DigitalCommons@University of Nebraska - Lincoln

2006

Direct detection of double-stranded DNA: molecular methods and applications for DNA diagnostics

Indraneel Ghosh

University of Arizona, ghosh@email.arizona.edu

Cliff I. Stains

University of Nebraska-Lincoln, cstains2@unl.edu

Aik T. Ooi

University of California - Davis

David J. Segal

University of California - Davis, djsegal@ucdavis.edu

Follow this and additional works at: https://digitalcommons.unl.edu/chemistrystains

Part of the Chemistry Commons

Ghosh, Indraneel; Stains, Cliff I.; Ooi, Aik T.; and Segal, David J., "Direct detection of double-stranded DNA: molecular methods and applications for DNA diagnostics" (2006). Cliff Stains Publications. 3.

https://digitalcommons.unl.edu/chemistrystains/3

This Article is brought to you for free and open access by the Published Research - Department of Chemistry at DigitalCommons@University of Nebraska - Lincoln. It has been accepted for inclusion in Cliff Stains Publications by an authorized administrator of DigitalCommons@University of Nebraska - Lincoln. 


\title{
Direct detection of double-stranded DNA: molecular methods and applications for DNA diagnostics $\dagger$
}

\author{
Indraneel Ghosh, ${ }^{* a}$ Cliff I. Stains, ${ }^{a}$ Aik T. Ooi ${ }^{b}$ and David J. Segal ${ }^{* b}$ \\ Received 2nd August 2006, Accepted 15th September 2006 \\ First published as an Advance Article on the web 28th September 2006 \\ DOI: $10.1039 / \mathrm{b} 611169 f$
}

Methodologies to detect DNA sequences with high sensitivity and specificity have tremendous potential as molecular diagnostic agents. Most current methods exploit the ability of singlestranded DNA (ssDNA) to base pair with high specificity to a complementary molecule. However, recent advances in robust techniques for recognition of DNA in the major and minor groove have made possible the direct detection of double-stranded DNA (dsDNA), without the need for denaturation, renaturation, or hybridization. This review will describe the progress in adapting polyamides, triplex DNA, and engineered zinc finger DNA-binding proteins as dsDNA diagnostic systems. In particular, the sequence-enabled reassembly (SEER) method, involving the use of custom zinc finger proteins, offers the potential for direct detection of dsDNA in cells, with implications for cell-based diagnostics and therapeutics.

\section{An introduction to DNA diagnostics}

Molecular diagnostics that report on DNA sequence information are making increasingly important contributions to

${ }^{a}$ Department of Chemistry, University of Arizona, Tucson, Arizona 85721 E-mail: ghosh@email.arizona.edu; Tel: 520-621-6331

${ }^{b}$ Genome Center and Department of Pharmacology, University of California, Davis, CA 95616 E-mail: djsegal@ucdavis.edu; Fax: 530-754-9658; Tel: 530-754-9134

$\uparrow$ Abbreviations: ZF, zinc finger; GFP, green fluorescent protein; PNA, peptide nucleic acid; dsDNA, double-stranded DNA; TFO, triplex-forming oligonucleotide; SNP, single nucleotide polymorphism; SEER, sequence-enabled reassembly. medicine and research. Pathogen identification based on a DNA sequence is more accurate, less subjective, and often much faster than culture-based methods. ${ }^{1,2}$ In addition to improving existing services, DNA diagnostics allow access to genomic information previously unavailable to clinicians. Genotyping methodologies, which reveal the status of the single nucleotide polymorphisms (SNP) that distinguish one individual's genome from another's, can provide insights into the most effective drug regimen for a particular patient, or provide clues to resistance or susceptibility for particular diseases. Such diagnostic information can usually be obtained by determining the presence, absence, or abundance of a

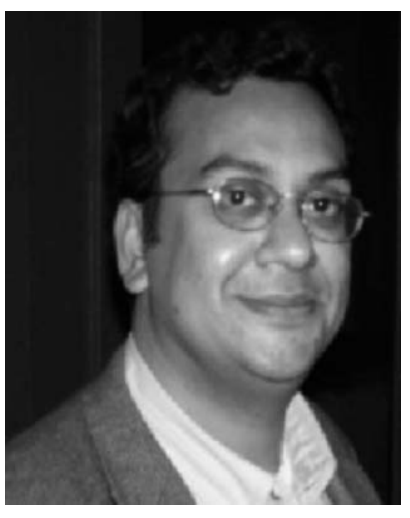

Indraneel Ghosh
Indraneel Ghosh was born on 30th December, 1968 in India. He obtained his BS degree in chemistry at Hobart College, Geneva, NY. He received his PhD in Chemistry at Purdue University, IN in 1998 with Professor Jean Chmielewski working on designing peptide inhibitors of protein-protein interactions and self-replicating peptides. Following his doctoral studies, Neel was a joint postdoctoral fellow at Yale University, New Haven, CT with Professor Andrew Hamilton in the Department of Chemistry and Professor Lynne Regan in Molecular Biophysics and Biochemistry. At Yale, he developed the split-Green Fluorescent Protein reporter system for the in vivo detection of protein-protein interactions. He started as an assistant professor at the University of Arizona, Tucson, AZ in 2001 and has been engaged in interdisciplinary research that spans the chemistry and biology interface. His current research interests are focused upon the design and selection of new proteins, peptides and small molecules that can be useful in the construction of therapeutics, biosensors, and biomaterials. He

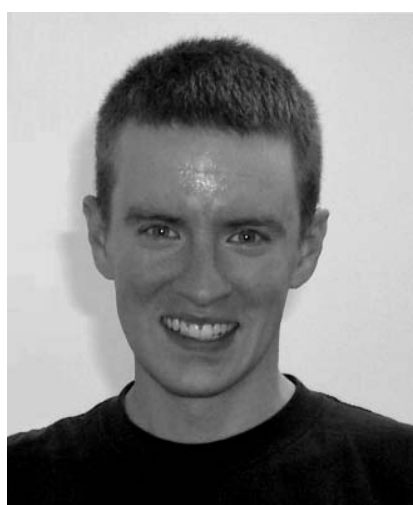

Cliff Stains has been a recipient of a Research Innovation Award from the Research Corporation and a Career Award from the National Science Foundation.

Cliff Stains was born in Lewistown, PA in 1979. He attended Millersville University, Millersville, $P A$ where he majored in Chemistry with an emphasis in Biochemistry. Under the direction of $\mathrm{Dr}$ Sandra Turchi he developed methods for the separation of methylated nucleotides, leading to an undergraduate honors thesis. Cliff joined the research group of Dr Indraneel Ghosh at the University of Arizona in 2003 where he is currently pursuing a PhD in Chemistry with a focus on Biological Chemistry. His research interests include designing protein-protein and protein-DNA assemblies for use in biological and materials applications. He has been the recipient of an institutional pre-doctoral Ruth L. Kirschstein National Research Service Award and mid-career awards at the University of Arizona. 
particular known sequence. This feature distinguishes such methods from general sequencing methodologies, which aim to determine large stretches of unknown sequence.

Many types of DNA diagnostic methodologies have been described. Some are in the very early stages of development while others are commercially available. One approach to categorize the myriad of techniques is to define how they address their common goals. Like all diagnostic technology, DNA diagnostics require both a detection method and a signal transducer. Most current detection methods for the sequencespecific recognition of DNA make use of the special property of single-stranded DNA (ssDNA) to base pair with high specificity to a complementary molecule (Fig. 1A). The other molecule may be another ssDNA, ssRNA, peptide-nucleic acid (PNA), ${ }^{3}$ or other base-pairing molecular analog. Such specific annealing or hybridization forms the basis for such common technologies as PCR amplification with specific primer sets, Southern blot, Northern blot, DNA microarray, and fluorescent in situ hybridization (FISH). ${ }^{4}$ However, there are other ways to read the sequence information besides Watson-Crick base-pairing (Fig. 1B). For example, polyamides are small chemical compounds that can be designed to bind with high sequence specificity in the minor groove of double-stranded DNA (dsDNA). ${ }^{5}$ Similarly, triplex-forming DNA, ${ }^{6}$ and zinc finger DNA-binding proteins ${ }^{7}$ can all be engineered to achieve specific base-pair recognition of dsDNA in the major groove.

The second component required is a signal transducer, which converts the sequence-specific recognition event into a signal that can be quantitatively measured (Fig. 1). Typically the signal is optical (colorimetric, fluorescent, luminescent, turbidic, etc) or electrical (voltage, resistance, or current change). Transducers with fluorescent readouts are used most commonly, such as dyes that intercalate into dsDNA (ethidium bromide and SYBR green) or are attached to the base-pairing

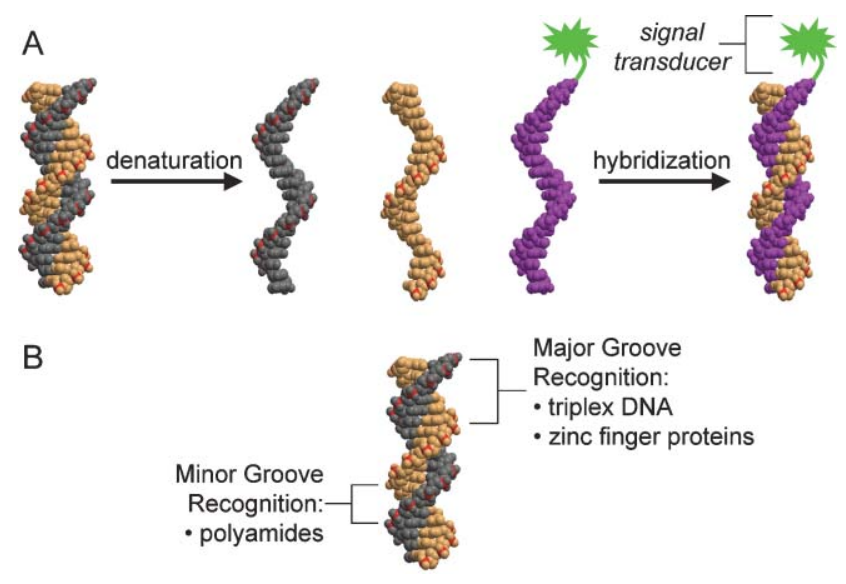

Fig. 1 An overview of DNA diagnostic methods. Detection methods can read the sequence information by either (A) Watson-Crick base pairing with one strand (orange), which requires denaturation of the duplex and subsequent hybridization with a complementary probe (purple), or (B) direct detection of dsDNA by specific interaction with base edges in the major or minor groove. A signal transducer converts the detection event into a quantitative signal, such as fluorescence intensity (green).

partner of ssDNA (labeled probes used in DNA microarrays, ${ }^{8}$ Taqman real-time PCR chemistry, ${ }^{9}$ or FISH). While the specificity of a DNA diagnostic will depend on the fidelity of the detection method, the sensitivity will largely be a function of the signal transducer. For example, PNA probes can be engineered to bind with extremely high specificity and affinity to their denatured chromosomal targets in a FISH assay. ${ }^{10}$ However, detection of unique genomic sequences is limited by the difficulty in detecting the weak signal of one fluorescent molecule over background. To improve sensitivity, several ingenious methods have recently been developed to sensitively

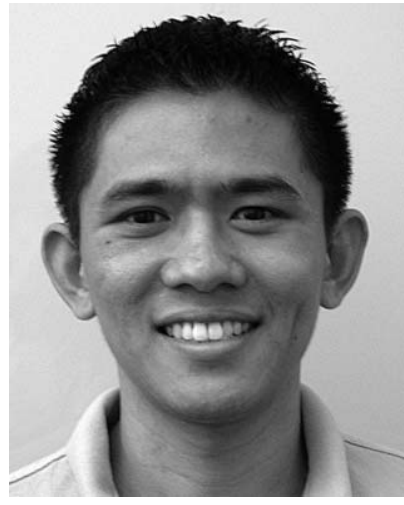

Aik Ooi
Aik Ooi was born in Penang, Malaysia in 1980. He obtained his BS degree in Chemistry at Mount Union College, Alliance, $\mathrm{OH}$, with a minor in Biology. For his undergraduate thesis, he worked under the supervision of Dr Laura Beal on the synthesis and application of substituted flavonoids as multi-drug resistant pump inhibitors in S. aureus. In 2002, Aik enrolled in the University of Arizona for his graduate studies in Medicinal and Natural Products Chemistry. He joined the research group of Dr David Segal, where he is working on the design and development of sequence-specific double-stranded DNA detection utilizing custom-designed zinc fingers and split-enzyme complementation. He has been the recipient of Yuma young investigator award and Caldwell research award at the University of Arizona.

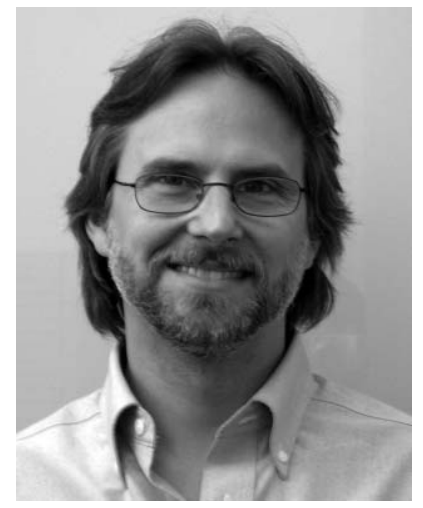

David Segal
David Segal was born in Yonkers, NY in 1966. He obtained a BS with honors in Biology from Cornell University, Ithaca, NY, in 1989. He obtained a PhD in Biochemistry from the University of Utah, Salt Lake City, UT, in 1996 with Professor Dana Carroll working on the stimulation of homologous recombination by targeting double-strand breaks in DNA. Seeking better targeting methods, he joined the laboratory of Professor Carlos Barbas at The Scripps Research Institute, La Jolla, CA as a postdoctoral fellow, where he helped develop the most widely used methods for engineering custom zinc finger DNA-binding proteins. As an assistant professor at the University of Arizona, Tucson, AZ, from 2002-2005, and now as an assistant professor at the University of California, Davis, CA, his research continues to focus on the design of engineered zinc fingers and their application as diagnostic agents and therapeutics. 
detect the recognition event (such as hybridization-dependent current fluctuations across an $\alpha$-hemolysin nanopore ${ }^{11,12}$ ), or amplify the transduced signal (such as hybridization-dependent release of barcode DNA from captured nanoparticles, ${ }^{13}$ or aggregation-enhanced fluorescence ${ }^{14}$ ). Some of these methods have proven to be extremely sensitive, able to detect molecules in the zeptomolar range (1-500 molecules per $\mathrm{ml}$ sample). Other strategies rely again on the special ability of nucleic acids to form specific base pairs and enzymatically amplify the DNA, either before or as part of the detection method (such as PCR, Strand Displacement Amplification (SDA) ${ }^{15}$ or Rolling Circle Amplification (RCA $)^{16}$ ).

For an overview of recent advances in hybridization-based DNA diagnostics, the reader is directed to several outstanding reviews on this topic. ${ }^{17-19}$ The scope of this review will be restricted to methods for the direct detection of dsDNA, meaning methods that do not require dsDNA denaturation and subsequent hybridization. Progress in this area has been slower because of the difficulty in engineering highly specific detection methods for the major or minor groove of DNA. However, the emergence of such technologies in the past decade has now enabled their application as dsDNA diagnostics. In some cases, the ability to use dsDNA as a substrate enables capabilities beyond what would be possible for hybridization-based methods.

\section{Beyond annealing: direct detection of dsDNA}

\section{Methods for direct detection of dsDNA in the minor groove}

Polyamides. Structural studies on the polyamide antibiotics, netropsin and distamycin A, demonstrated their sequencespecific minor groove targeting capabilities. ${ }^{20}$ Dickerson and Wemmer have demonstrated that distamycin A is capable of binding as a homodimer in the minor groove by specifically targeting AT-rich sequences. Building on these initial observations and by further synthetic, structural and biophysical studies, Dervan and coworkers have developed a powerful and general approach for the sequence-specific recognition of the minor groove of dsDNA utilizing designed hairpin polyamides, composed of $N$-methylpyrroles and
$N$-methylimidazoles (Fig. 2). 5,21,22 These seminal studies have allowed polyamides to emerge as a useful molecular recognition tool kit for sequence-specific targeting of dsDNA.

Laemmli and coworkers have recently utilized fluoresceinlabeled polyamides as "chromosome paints" with the goal to visualize AT-rich satellite regions and scaffold-associated regions in the genome of Drosophila melanogaster. ${ }^{23}$ In a second study, designed polyamides conjugated to Texas-Red were used to target and visualize telomeric repeats in insects (TTAGG) and vertebrates (TTAGGG) with high specificity. ${ }^{24}$ These results demonstrated that telomere-specific polyamidedye conjugates might allow for the rapid estimation of the telomere length. These elegant studies utilized fluorescence microscopy of fixed cells or of isolated nuclei, where excess labeled-polyamides can be removed. However, the detection of dsDNA in live cells or whole animals would require a method for removing unbound labeled-polyamides, as the background fluorescence would likely decrease the contrast.

With the goal of lowering background (signal from unbound labeled-polyamides), Dervan and coworkers have recently designed and tested polyamides conjugated to intercalating dyes tetramethyl rhodamine and thiazole orange (Fig. 2, right). ${ }^{25,26}$ In these studies, several fluorescent conjugates were synthesized and tested against dsDNA targets, 5'-WGGGWW-3', 5'-WGGCCW-3', and 5'-WGWWCW-3' $(\mathrm{W}=\mathrm{A}$ or $\mathrm{T})$. It was found that the designed conjugates with thiazole orange exhibit $>1000$-fold fluorescence enhancement only in the presence of specific target dsDNA, where the dye likely intercalates at an adjacent site. The lowest concentration of oligonucleotide detected in this study was $1 \mathrm{nM}$, although lower concentrations were not examined. Mismatched targets reduced the signal by $>90 \%$. As polyamide binding site sizes and sequence specificities are being further refined, these new dsDNA-sensitive dyes attached to appropriate targeting molecules will likely find use in probing dsDNA in a cellular setting. $^{27}$

\section{Methods for direct detection of dsDNA in the major groove}

Triplex DNA. Triple helix-forming oligonucleotides (TFO) bind to polypurine/polypyrimidine tracts in the major groove
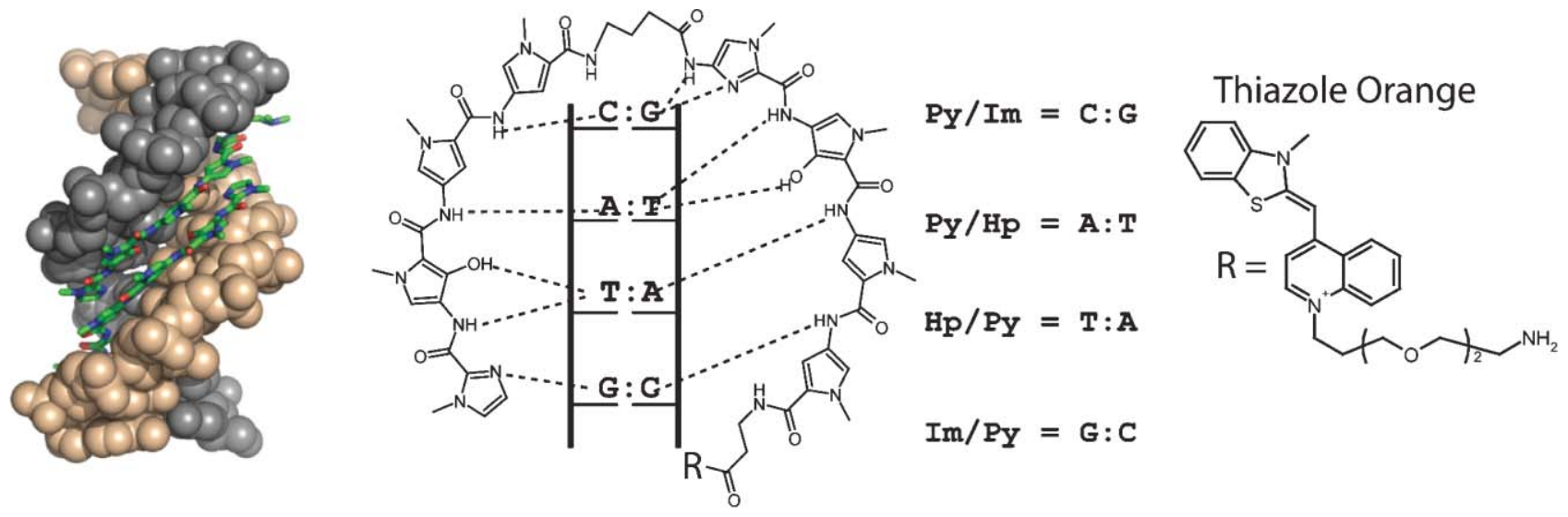

Fig. 2 Polyamide minor groove binders. Left: structural representation of a polyamide (green) bound in the minor groove of dsDNA (black and orange). Middle: a schematic illustration of the binding interactions. The abbreviations are pyrrole (Py), hydroxypyrrole (Hp), and imidazole (Im). Right: chemical structure of thiazole orange, used as a signal transducer. 

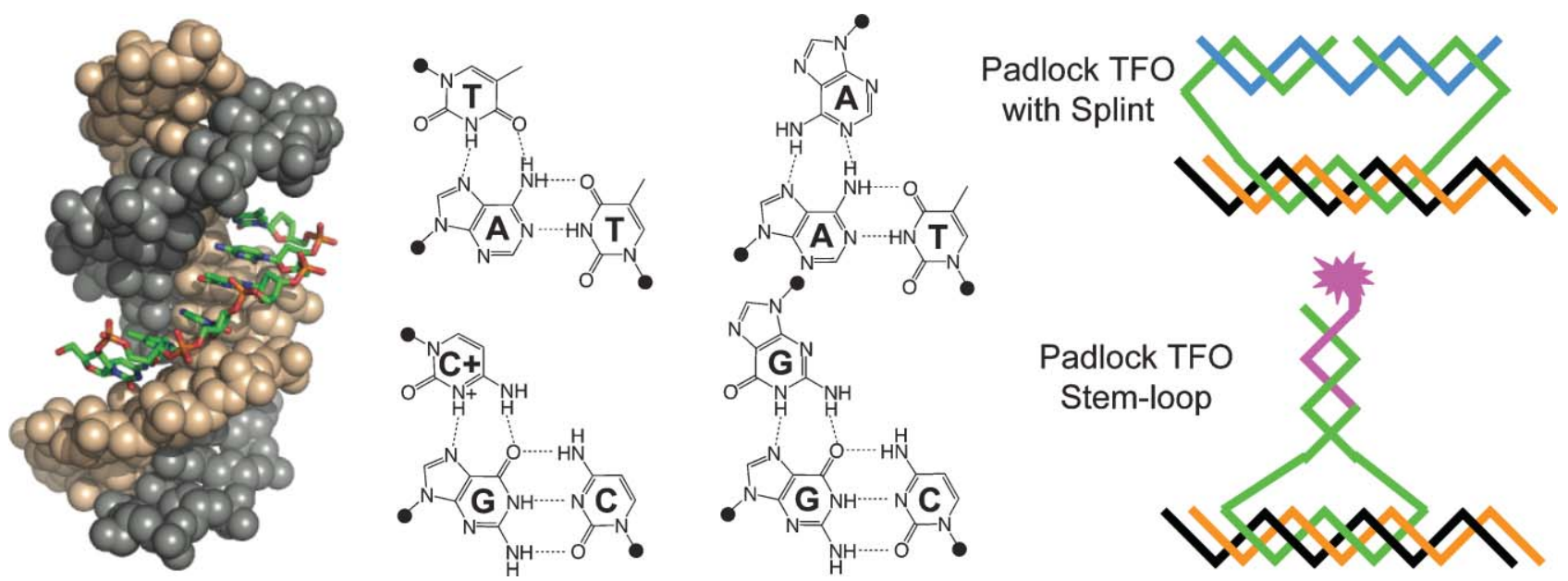

Fig. 3 Triplex-DNA major groove binders. Left: structural representation of a TFO (green) bound in the major groove of dsDNA (black and orange). Middle: a schematic illustration of the binding interactions. Right: two types of "padlock" TFO strategies, (top) linear TFO with ends joined using a "splint" oligonucleotide (blue), and (bottom) stem-loop TFO with ligated fluorescently labeled DNA (purple).

of dsDNA (Fig. 3). ${ }^{28,29}$ Sequence-specific recognition is achieved by Hoogsteen or reverse Hoogsteen hydrogen bonding to purines in the major groove, thus restricting triplex formation to sequences with purines on one strand. ${ }^{6}$ TFO composed of polypurines bind in a direction antiparallel to the purine strand of the duplex. However, G-rich TFO are susceptible to forming competing G-quadruplex structures. ${ }^{30}$ TFO composed of polypyrimidines bind parallel to the purine strand, however the cytosines must be protonated. The binding of polypyrimidine TFO is therefore $\mathrm{pH}$ dependent, and does not readily occur at physiological $\mathrm{pH}$. 5-Methylcytosine is often substituted for cytosine to improve affinity, but does not avoid the requirement for protonation. TFO composed of thymine and guanine bases are also frequently used. Unlike polyamides, it is fairly trivial to make a 16-20 nt TFO, which should have sufficient specificity to target a unique site in the human genome.

An early application of triplex-DNA as a diagnostic agent was to stain an alpha-satellite repeat in chromosomal spreads using an assay analogous to FISH (appropriately termed TISH). ${ }^{31}$ A $16 \mathrm{nt}$ polypyrimidine TFO was designed to bind the 500-1000 repeats of the target sequence at $\mathrm{pH} 6$ without denaturation. TFO binding was stabilized by crosslinking to the duplex via a tethered psoralen moiety. Signal transduction was accomplished by tagging the TFO with fluorescein isothiocyanate (FITC). The study found TISH comparable to FISH in both sensitivity and specificity. The dsDNA-based TISH was suggested to be more quantitative than FISH, since there was no competition between probe hybridization and duplex reannealing. Additionally, non-denatured DNA allowed TISH, but not FISH, to be compatible with G-banding chromosomal reference techniques.

Another application of triplex DNA is the so called "padlock" TFO. ${ }^{32}$ The detection method is based on the central part of a linear TFO, which forms a triplex with the target duplex DNA. The ends of the linear molecule can be joined covalently by base-pairing with an additional "splint" oligonuclotide, followed by DNA ligase (Fig. 3, top right). The result is a circular ssDNA molecule that is topologically linked to the target duplex. Signal transduction can be accomplished by $\mathrm{RCA},{ }^{16}$ or other amplification method. The ends of the TFO can alternatively be stabilized non-covalently by a stemloop structure (Fig. 3, bottom right). ${ }^{33,34}$ Signal transduction in this case can be accomplished by designing the stem loop to have a short terminal overhang, to which a fluorescently labeled DNA can be ligated. Because the TFO is physically wrapped around the duplex molecule (hence the name "padlock"), the affinity of this complex is far greater than that of the triplex alone. In one study, a polypurine/ polypyrimidine tract in individually spread molecules of lambda phage DNA was visualized with a $59 \mathrm{nt}$ stem-loop oligonucleotide probe (containing a $15 \mathrm{nt}$ central triplex forming region) and a $500 \mathrm{bp}$ stem-loop-binding labeled duplex. ${ }^{33}$ For increased sensitivity, the 500 bp DNA was labeled with at least 20 molecules of AlexaFluor 546. The purified lambda DNA was stretched on glass slides using molecular combing methods. The sample had to be heated to unwind the stem-loop on the probe oligonucleotide, then slowly cooled to allow rewinding after triplex formation. The precise position of the target site along individual DNAs was easily observable by fluorescence microscopy. In another study, radiolabeled padlock TFO were able to detect subfemtomolar concentrations of target dsDNA using a signal transduction method of gel electrophoresis followed by autoradiography of dried gels. ${ }^{34}$

A wide variety of technological improvements have been made to expand recognition beyond strictly polypurine tracts, improve affinity, reduce $\mathrm{pH}$ dependence, and reduce degradation in cells. For example, artificial base analogs can extend recognition to all possible base pairs. ${ }^{35}$ Chemical compounds such as BQQ can act as triplex stabilizing agents. ${ }^{36}$ Triplex formation involving any sequence of bases was suggested to occur at physiological $\mathrm{pH}$ in the presence of YOYO-1, an oxazole yellow homodimer the fluorescent intensity of which increases over 1000 -fold in the presence of dsDNA and 100000 -fold in the presence of triplex DNA. ${ }^{37}$ This approach was reported to distinguish SNPs and single base pair deletions in PCR amplified fragments of cystic fibrosis gene, the DNA 
repair gene $h M S H 2$, and the tumor suppressor genes $B R C A 1$ and $P 16$. The assay was extremely rapid and simple; however, the postulated triplexes were not unequivocally demonstrated. Such modified triplex paradigms and their general applicability for direct dsDNA recognition deserve further study.

Other triplex-like detection methods. Various other dsDNA detection methods involve both interactions in the major and/ or minor groove as well as Watson-Crick base pairing interactions accompanied by strand displacement. Two examples of such methods are RecA- and PNA-mediated dsDNA complexes. The E. coli recombination protein RecA catalyzes a homology-dependent strand invasion and exchange reaction in which the invading strand ultimately becomes base-paired to the complementary duplex strand. The initial recognition of the homologous region is based on a triplex structure. ${ }^{38,39}$

Peptide nucleic acid (PNA) are synthetic nucleic acid homologs containing standard DNA bases but a polyamide backbone composed of $N$-(2-aminoethyl) glycine units. The nucleobases are attached with methylenecarbonyl linkers. ${ }^{40}$ The neutral backbone eliminates the charge repulsion in standard nucleic acid hybridization, therefore PNA is able to bind DNA and RNA with extremely high affinity and specificity. In the most common application, a homopyrimidine PNA forms a structure in which one PNA molecule forms Watson-Crick base pair interactions with a polypurine DNA strand, while another PNA forms Hoogsteen interactions. The other DNA strand is displaced, forming a "P loop". However, a variety of PNA:DNA $2, \mathrm{PNA}_{2}$ :DNA, $\mathrm{PNA}_{2}$ :DNA 2 structures have been observed under various experimental conditions. ${ }^{3}$

DNA detection applications have been developed using both RecA $^{41}$ and PNA. ${ }^{42-44}$ Indeed, applications of PNA are so numerous that the reader is directed to a dedicated review on this subject. ${ }^{45}$ However, as both these technologies involve some aspect of local duplex disruption and are not strictly major or minor groove detection methods, they will not be discussed further here.

Sequence-specific DNA-binding proteins. The most common method for dsDNA recognition found in nature is the use of sequence-specific DNA-binding proteins. The various transcription factors, repair proteins, and DNA maintenance enzymes (nucleases, topoisomerases, helicases) comprise one of the largest functional groups encoded in the human genome. ${ }^{46}$ Several proof-of-concept experiments explored the coupling of the dsDNA detection capabilities of natural DNAbinding proteins with a signal transduction system. In a study similar to the polyamide experiments described earlier, a 50 residue peptide corresponding to the DNA-binding domain of Hin recombinase was attached to the dye oxazole-yellow, allowing for fluorescence enhancement only in the presence of target dsDNA. ${ }^{47}$ In another study, the site-specific restriction enzyme EcoRI was conjugated to $20 \mathrm{~nm}$ fluorescent nanoparticles. ${ }^{48}$ In the absence of magnesium ions, EcoRI was able to bind its target without subsequent cleavage. In an assay similar to the padlock TFO described earlier, the EcoRInanoparticle conjugates enabled direct visualization of EcoRI sites on stretched single DNA molecules using fluorescence microscopy.
Beyond in vitro assays, the E. coli lac repressor was fused to green fluorescent protein (GFP) to visualize inserted repeats of the lac operator in living yeast and mammalian cells. ${ }^{49}$ Single chromosomal integrations of a vector containing 256 repeats of the lac operator could be observed by fluorescence microscopy in cells expressing the GFP-lac fusion protein, with a signal-to-noise (background nuclear fluorescence) ratio of $12: 1$. In the absence of DNA binding, the expression of GFP-lac produced only diffuse fluorescence. The sensitivity of this live cell imaging method was found to be comparable to immunostaining and FISH. The study went on to demonstrate the utility of this method to examine chromonema fibers in interphase nuclei. The authors noted that previous failures to appreciate large-scale chromatin substructure within chromosome domains were consistent with structural perturbations in chromatin structure resulting from standard in situ hybridization procedures. More recently, the GFP-lac system was used to perform mosaic analysis in living C. elegans. ${ }^{50}$

The GFP-lac studies demonstrate successful detection in an environment in which the direct detection of dsDNA is a clear advantage, the living cell. However, these studies also illustrate the technological challenges to advancing beyond the proof-ofconcept stage. First, the use of natural DNA-binding proteins as the detection method severely restricts the spectrum of DNA sequences that can be recognized. Second, signal transduction was successful because 256 GFP-lac molecules were spatially restricted to one locus. Detection of spatially distributed or unique target sequences, as is more typically desired, would be impossible because the individual bound GFP-lac molecules would have the same signal intensity as unbound molecules. We have attempted to address both of these challenges in the recently described sequence-enabled reassembly (SEER) detection methodology. ${ }^{51-53}$ As will be described below, the detection method is based on engineered zinc finger proteins, the specificity of which can be programmed by the investigator. The signal transducer is based on the binding-dependent reassembly of a reporter protein, such that no signal should be present unless DNA-binding occurs.

\section{The SEER method for direct detection of dsDNA}

Engineered zinc finger DNA-binding proteins. $\mathrm{Cys}_{2}-\mathrm{His}_{2}$ zinc finger domains are the largest family of DNA binding domains in the human genome. ${ }^{46}$ Each domain is a small peptide consisting of 30 amino acids folded into a $\beta \beta \alpha$ structure that is stabilized by the chelation of a zinc ion to the two cysteine and two histidine residues. ${ }^{54}$ The specificity of zinc fingers is determined by the $\alpha$-helix region, which is inserted into the major groove of DNA where it contacts 3 or 4 bases. These domains can exist as tandem repeats to form multi-finger structures recognizing extended DNA sequences (Fig. 4). Zinc fingers are highly specific and normally bind to DNA with nanomolar to picomolar affinity.

Using phage display technology, DNA contacting amino acids in the zinc finger domain were randomized for the selection of new variants that recognize desired DNA sequences. $^{55,56}$ This allowed the selection of modules to construct multi-domain zinc fingers to bind to specific DNA sequences. Currently, domains have been identified that 


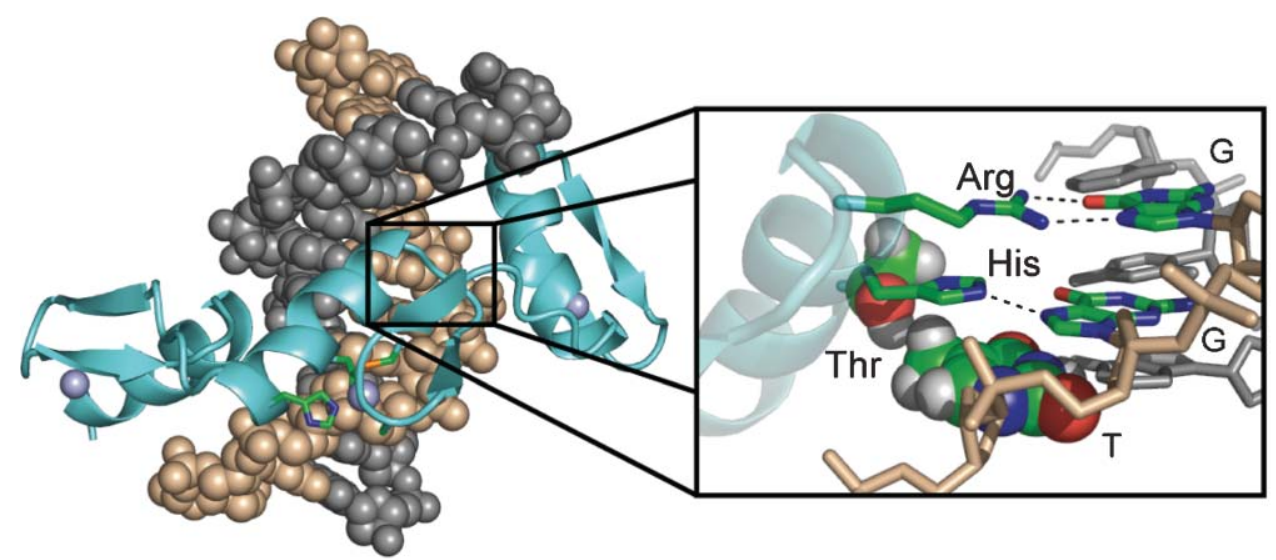

\begin{tabular}{|c|c|c|c|c|c|}
\hline $\begin{array}{l}\text { Triplet } \\
\text { (ANN) }\end{array}$ & $\begin{array}{c}\text { Module } \\
-112-3-456 \\
\end{array}$ & $\begin{array}{l}\text { Triplet } \\
\text { (CNN) }\end{array}$ & $\begin{array}{c}\text { Module } \\
-112-3-456 \\
\end{array}$ & $\begin{array}{l}\text { Triplet } \\
\text { (GNN) }\end{array}$ & $\begin{array}{c}\text { Module } \\
-112-3-456 \\
\end{array}$ \\
\hline 5'-AAA-3' & QRA-N-LRA & 5'-CAA-3' & QSG-N-LTE & 5'-GAA-3' & QSS-N-LVR \\
\hline 5'-AAC-3' & DSG-N-LRV & 5'-CAC-3' & SKK-A-LTE & 5'-GAC-3' & DPG-N-LVR \\
\hline 5'-AAG-3' & RKD-N-LKN & 5'-CAG-3' & RAD-N-LTE & 5'-GAG-3' & RSD-N-LVR \\
\hline 5'-AAT-3' & TTG-N-LTV & 5'-CAT-3' & TSG-N-LTE & 5'-GAT-3' & TSG-N-LVR \\
\hline 5'-ACA-3' & SPA-D-LTR & 5'-CCA-3' & TSH-S-LTE & 5'-GCA-3' & QSG-D-LRR \\
\hline 5'-ACC-3' & DKK-D-LTR & $5^{\prime}-\mathrm{CCC}-3^{\prime}$ & SKK-H-LAE & 5'-GCC-3' & DCR-D-LAR \\
\hline 5'-ACG-3' & RTD-T-LRD & 5'-CCG-3' & RND-T-LTE & 5'-GCG-3' & RSD-D-LVR \\
\hline 5'-ACT-3' & THL-D-LIR & 5'-CCT-3' & TKN-S-LTE & 5'-GCT-3' & TSG-E-LVR \\
\hline 5'-AGA-3' & QLA-H-LRA & $5^{\prime}-\mathrm{CGA}-3^{\prime}$ & QSG-H-LTE & 5'-GGA-3' & QRA-H-LER \\
\hline 5'-AGC-3' & N/A & 5'-CGC-3' & HTG-H-LLE & 5'-GGC-3' & DPG-H-LVR \\
\hline 5'-AGG-3' & RSD-H-LTN & 5'-CGG-3' & RSD-K-LTE & 5'-GGG-3' & RSD-K-LVR \\
\hline 5'-AGT-3' & HRT-T-LTN & 5'-CGT-3' & SRR-T-CRA & 5'-GGT-3' & TSG-H-LVR \\
\hline 5'-ATA-3' & QKS-S-LIA & 5'-CTA-3' & QNS-T-LTE & 5'-GTA-3' & QSS-S-LVR \\
\hline 5'-ATC-3' & N/A & 5'-CTC-3' & N/A & 5'-GTC-3' & DPG-A-LVR \\
\hline 5'-ATG-3' & RRD-E-LNV & 5'-CTG-3' & RND-A-LTE & 5'-GTG-3' & RSD-E-LVR \\
\hline 5'-ATT-3' & HKN-A-LQN & 5'-CTT-3' & TTG-A-LTE & 5'-GTT-3' & TSG-S-LVR \\
\hline
\end{tabular}

Fig. 4 Zinc finger protein major groove binders. Top: structural representation of a three zinc finger protein (blue) bound in the major groove of dsDNA (black and orange). Bottom: recognition modules incorporated into the alpha helix of a zinc finger that will enable it to specifically bind the indicated $5^{\prime}-\mathrm{ANN}-3^{\prime}, 5^{\prime}-\mathrm{CNN}-3^{\prime}$ or 5'-GNN-3' DNA sequence.

facilitate binding to all $5^{\prime}-\mathrm{GNN}-3^{\prime}$, most $5^{\prime}-\mathrm{ANN}-3^{\prime}$ and $5^{\prime}-\mathrm{CNN}-3^{\prime}$, and some $5^{\prime}-\mathrm{TNN}-3^{\prime}$ type sequences, enabling targeting to an extremely wide spectrum of target sites. ${ }^{57-59}$ Multi-finger proteins based on these custom DNA binding domains can be assembled by PCR using overlapping oligonucleotides or commercial synthesis. ${ }^{60,61}$

SEER-GFP. Simply attaching a fluoresent molecule such as GFP to an engineered zinc finger protein would probably generate a useful reagent for visualizing repeated sequences. Such a reagent would be similar to the polyamide paintbrush, TISH, or GFP-lac fusions described above, and could be targeted to biologically relevant repeated elements. However, to visualize low copy number or unique sequences would require a mechanism to reduce the background, that is, to eliminate fluorescence in the absence of DNA binding. Sequence-enabled reassembly of GFP (SEER-GFP) ${ }^{53}$ reduces background through the convergence of custom-designed zinc fingers and a split-protein system ${ }^{62}$ to create a "turn-on" DNA biosensor (Fig. 5A). In the split-protein system, the GFP was dissected into two fragments that were non-fluorescent, and would not assemble to form the functional protein by themselves without the assistance of dimerization domains. ${ }^{63}$ In SEER-GFP, the GFP fragments were fused to zinc fingers via a 15 amino acid peptide linker. The hypothesis was that in the presence of adjacent DNA sequences recognized by the zinc fingers, the GFP fragments would be brought into proximity for refolding into a functional fluorophore upon DNA binding. The two proteins were purified and the equimolar mixtures were incubated in the absence and presence of oligonucleotide containing the target sites of the zinc fingers separated by $10 \mathrm{bp}$. Fluorescent spectra were obtained after $48 \mathrm{~h}$, showing functional GFP only in the presence of target oligonucleotides. No fluorescence was detected when only one of the proteins was present, or when the proteins were mixed with non-specific herring sperm DNA.

SEER-LAC. Sequence-enabled reassembly of $\beta$-lactamase (SEER-LAC) was a modified version of SEER-GFP, such that the GFP fragments were replaced by the antibiotic-resistant 

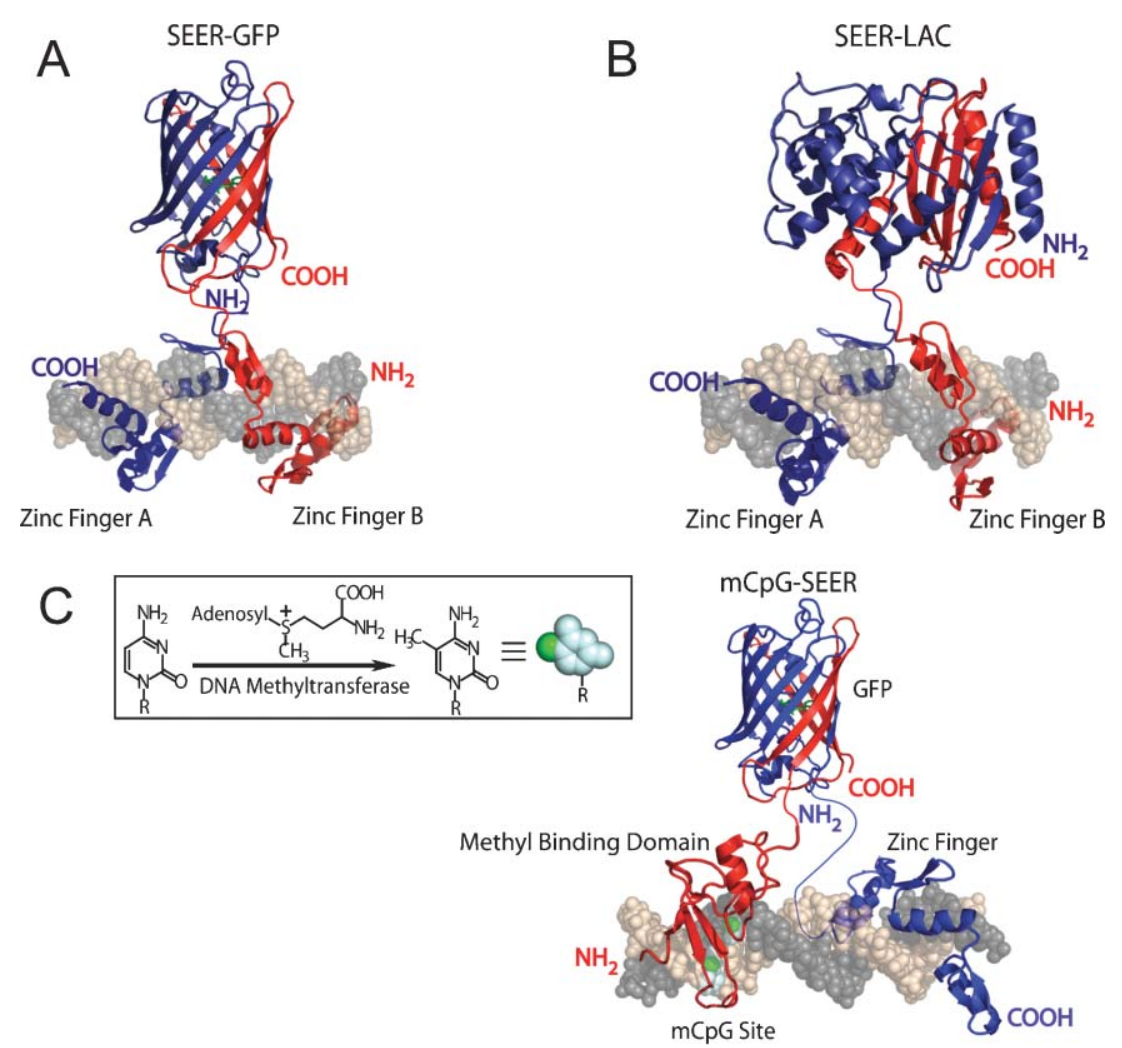

Fig. 5 The SEER method for the direct detection of dsDNA. (A) SEER-GFP, (B) SEER-LAC, (C) mCpG-SEER with GFP.

enzyme TEM-1 $\beta$-lactamase (Fig. 5B). ${ }^{51}$ This enzyme had several attractive features for our studies. Because eukaryotic cells do not contain an endogenous $\beta$-lactamase activity, a signal generated from this enzyme was expected to have little background. Its activity could be monitored both in vitro, using the chromogenic substrate nitrocefin, and in vivo, using the fluorescent substrates such as CCF2/AM or CC2.${ }^{64}$ Like GFP, fragments of TEM-1 $\beta$-lactamase had been generated that could reassemble when attached to appropriate dimerization domains. ${ }^{65}$ The constructs of SEER-LAC were similar to that of SEER-GFP, where the lactamase fragments were linked to zinc fingers via a 15 amino acid linker. SEER-LAC required substrate to produce a signal when the correct DNA sequences were present, making it possible to amplify the intensity of the signal. This enzymatic system was a 1000 -fold improvement in detection time over SEER-GFP, as it could differentiate target from non-target DNA sequences in 5 min. The colorimetric assay format had sufficient sensitivity to easily detect $20 \mathrm{nM}$ of purified target DNA. The specificity of the system was high enough to distinguish a single base-pair mutation in the $18 \mathrm{bp}$ binding site. The intensity of the signal remained the same in the presence of equal mass herring sperm DNA to target oligonucleotide.

mCpG-SEER: detection of chemically modified DNA. Beyond the catalogue of genes and their products in the human genome, there exists a second level of complexity comprising the carefully regulated chemical modification of DNA and its associated proteins. DNA methylation plays a central role in the epigenetic modification of genomic DNA and directly regulates transcription and chromatin structure. ${ }^{66-68}$ The methylation of cytosine at the $5^{\prime}$-position in humans is the only documented epigenetic modification, which is controlled by DNA methyltransferases, methyl-CpG binding proteins, and a postulated DNA demethylase. Standard PCR amplification of DNA from tissue samples, followed by either sequencing or microarray detection cannot distinguish between cytosine and methylated cytosine. The detection of methylated $\mathrm{CpG}$ sites was revolutionized by the bisulfite modification technique that converts unmethylated cytosine to uracil but not methylated cytosine, thus allowing for discrimination between methylated versus unmethylated sites. ${ }^{69,70}$ Bisulfite-modified DNA can be subsequently amplified by PCR and analyzed by conventional sequencing or microarray methods. Alternatively, bisulfite-modified DNA can be analyzed by methylation-specific PCR in which appropriate primers are designed to selectively recognize and amplify unmodified CpG-containing sites. The bisulfite method requires denaturing double-stranded DNA and bisulfite treatment for $4-18 \mathrm{~h}$, followed by sequencing, microarray detection, or methylation-specific $\mathrm{PCR}^{71}$ and their variations. ${ }^{72}$ Some problems arising from these approaches are: (a) DNA occasionally partially degrades ${ }^{73}$ (b) incomplete bisulfite reactions result in false positives $;{ }^{74}$ (c) resulting singlestranded DNA adopts alternate folded conformations ${ }^{75}$ that prevent PCR amplifications; (d) primer design becomes problematic in methylation-specific PCR; and (e) microarray detection technologies are expensive. ${ }^{76}$ Many of the above artifacts can be solved ${ }^{77}$ with appropriate changes in experimental conditions, however the total time for this reaction and 
analysis is long and remains constant. Thus, an alternate approach, independent of bisulfite treatment and PCR amplification, that could rapidly detect the methylation status of $\mathrm{CpG}$ islands in known promoter sites would be of considerable benefit in this area.

Towards the goal of a rapid method for the detection of known sites of hypermethylation at $\mathrm{CpG}$ islands, we have recently developed a new approach called $\mathrm{mCpG-SEER}$ (Fig. 5C). ${ }^{52}$ The $\mathrm{mCpG}-\mathrm{SEER}$ system was designed based upon our existing SEER-GFP system, while incorporating a means for targeting methylated $\mathrm{CpG}$ sites. We chose the wellcharacterized MBD2 protein from humans, that has a binding affinity of $2.7 \mathrm{nM}$ for $\mathrm{mCpG}$ sites while it has a $50-100$ fold reduced binding affinity for unmethylated $\mathrm{CpG}$ sites. We hypothesized that this difference in binding affinities would allow us to selectively target $\mathrm{mCpG}$ sites versus unmethylated $\mathrm{CpG}$ sites. Since numerous sites on a genome are methylated, we needed to introduce sequence selectivity, which can be readily achieved by utilizing natural and designed zinc fingers as discussed. As proof of concept, we employed the Zif268 zinc finger to recognize a site next to the $\mathrm{mCpG}$ site. We found that the specificity of $\mathrm{mCpG-SEER}$ was $>40$-fold between a methylated versus a non-methylated $\mathrm{CpG}$ target site. We also found that the fluorescent signal was linear to $5 \mathrm{pmol}$ of methylated target DNA in a $100 \mu \mathrm{L}$ sample volume. Thus, mCpG-SEER represents a new and potentially useful method for the direct detection of $\mathrm{CpG}$ methylation, which may find numerous applications in delineating the epigenome and in cancer research.

\section{Current status and future of direct detection methods for dsDNA}

\section{Why is direct detection of dsDNA important?}

DNA is rarely present in single-stranded form, either naturally or after PCR amplification. Improvements in the detection of either type of dsDNA should lead to more robust and flexible DNA diagnostics. The dsDNA-based TISH was suggested to be more quantitative than FISH, since there was no competition between probe hybridization and duplex reannealing. ${ }^{31}$ Also, perturbations in chromatin structure may result from standard in situ hybridization procedures. ${ }^{49}$ Several of the assays described were extremely fast and simple, requiring no duplex denaturation or careful control of temperature. Conceivably, such methods could reduce assay time as well as the costs associated with sophisticated instrumentation and highly-trained technicians.

\section{Into the cell}

Direct detection of dsDNA would also have an obvious advantage for the visualization of genomic information in living cells. Methods for determining genotype, chromatin status, and target copy number in individual living cells have been largely inaccessible using currently available hybridization-based techniques. ${ }^{78}$ Triplex DNA, polyamides and engineered zinc finger proteins have all been used in cells with some efficacy as gene regulators ${ }^{79}$ and inducers of DNA damage or homologous recombination. ${ }^{80}$ Therefore, they are good candidates for cellular diagnostic assays, although none have yet been evaluated in this role. Table 1 compares several features of the methods described in this review. Methods such as fluorescein-conjugated polyamides or triplex (TISH) could be applied to visualize repeated regions in living cells. Background fluorescence would be expected to be high, but the GFP-lac study suggests binding to spatially restricted loci might generate sufficient signal. Methods such as Thiazole orange-polyamides or SEER should have less background, and therefore should be even more sensitive. However, the sensitivity of all these methods will likely need to be improved in order to detect unique sequences in cells, such as SNPs, translocations, or mutations.

\section{Beyond detection}

While any of these methods might eventually be suitable for dsDNA detection, SEER offers several additional capabilities. The ability to use other types of DNA-binding domains enables the recognition of other types of information, such as DNA methylation (mCpG-SEER), adducts and damage. In cells, the ability to reassemble enzymatic functions in response to genotype could have applications for gene therapy. Also, the use of cytotoxic substrates ${ }^{81}$ or enzymes in cells could

Table 1 Methods for direct detection of dsDNA

\begin{tabular}{|c|c|c|c|c|c|}
\hline Method & Assay & Sensitivity & Sequence restrictions & Likely to be useful in cells & Reference \\
\hline \multicolumn{6}{|l|}{ Polyamide: } \\
\hline Fluorescein conjugate & FISH-like & Highly repeated sequences & None & Yes & 23,24 \\
\hline Thiazole orange & Oligo targets & $1 \mathrm{nM}^{a}$ & None & Yes & 25 \\
\hline \multicolumn{6}{|l|}{ Triplex: } \\
\hline TISH & FISH-like & Highly repeated sequences & Polypurine tracts & Yes & 31 \\
\hline Padlock-FITC & Spread molecules & Single molecule & Polypurine tracts & No, heat requirement & 33 \\
\hline Padlock-radiolabeled & Southern & $<1 \mathrm{fM}$ & Polypurine tracts & No, heat requirement & 34 \\
\hline YOYO-1 & Amplified DNA & $4 \mathrm{nM}^{a}$ & None & No, intercalator & 37 \\
\hline \multicolumn{6}{|l|}{ Protein: } \\
\hline Hin-oxazole yellow & Oligo targets & $50 \mathrm{nM}^{a}$ & Hin sites & Delivery? & 47 \\
\hline EcoRI-nano & Spread molecules & Single molecule & EcoRI sites & No, cleavage & 48 \\
\hline $\mathrm{GFP}-l a c$ & Live cell & 256 tandem repeats & lac sites & Yes & 49 \\
\hline SEER-GFP & Oligo targets & $2.5 \mu \mathrm{M}^{a}$ & Few & Yes & 53 \\
\hline SEER-LAC & Oligo targets & $20 \mathrm{nM}$ & Few & Yes & 51 \\
\hline mCpG SEER & Oligo targets & $50 \mathrm{nM}$ & Few & Yes & 52 \\
\hline
\end{tabular}


enable sequence-dependent cell killing, with applications for therapeutics.

\section{Conclusions}

If the field of DNA diagnostics is in its infancy, methods for the direct detection of dsDNA are embryonic. Most of the recent work is still proof-of-concept experiments. This is partially technological. TFO have been used since the 1980s, polyamides were developed in the mid 1990s and the first generally accessible methods for engineering zinc finger proteins were described in 1999. Polyamides have the least sequence recognition restrictions, but are generally limited to short (i.e. multiple) target sites. TFO can recognize long (i.e. unique) target sites, but are generally limited to polypurine tracts. Engineered zinc finger proteins can be designed to long target sites with less sequence restrictions, but are still currently unable to target all possible sequences. However, the pace and scope of new investigations continued to increase, and some promising candidate methods (such as triplex with YOYO-1) are already entering commercial development. The field is benefiting from intensifying research in other applications of dsDNA recognition technology, primarily in the areas of gene regulation and gene disruption/correction. There is also a growing consensus to bridge the gap between the basic scientists who develop these methods but are unfamiliar with their applications, and the clinicians who would use these methods but are unfamiliar with the technological challenges. The greatest progress will certainly be made by the collaboration between these two camps.

\section{Acknowledgements}

C.I.S. was supported by an NIH training grant. D.J.S. and I.G. were supported by GM077403 and CA122630. We thank Bogdan Olenyuk for helpful comments on this manuscript.

\section{References}

1 S. D. Atkins and I. M. Clark, J. Appl. Genet., 2004, 45, 3-15.

2 M. M. Lopez, E. Bertolini, A. Olmos, P. Caruso, M. T. Gorris, P. Llop, R. Penyalver and M. Cambra, Int. Microbiol., 2003, 6, 233-243.

3 P. E. Nielsen, Curr. Med. Chem., 2001, 8, 545-550.

4 J. M. Levsky and R. H. Singer, J. Cell Sci., 2003, 116, 2833-2838.

5 P. B. Dervan, Bioorg. Med. Chem., 2001, 9, 2215-2235.

6 P. P. Chan and P. M. Glazer, J. Mol. Med., 1997, 75, 267-282.

7 D. Segal and C. F. Barbas III, Curr. Opin. Biotechnol., 2001, 12, 632-637.

8 J. D. Hoheisel, Nat. Rev. Genet., 2006, 7, 200-210.

9 F. M. De la Vega, K. D. Lazaruk, M. D. Rhodes and M. H. Wenz, Mutat. Res., 2005, 573, 111-135.

10 H. Stender, Expert. Rev. Mol. Diagn., 2003, 3, 649-655.

11 S. Howorka, S. Cheley and H. Bayley, Nat. Biotechnol., 2001, 19, 636-639.

12 W. Vercoutere, S. Winters-Hilt, H. Olsen, D. Deamer, D. Haussler and M. Akeson, Nat. Biotechnol., 2001, 19, 248-252.

13 J. M. Nam, S. I. Stoeva and C. A. Mirkin, J. Am. Chem. Soc., 2004, 126, 5932-5933.

14 K. Dore, M. Leclerc and D. Boudreau, J. Fluoresc., 2006, 16, 259-265.

15 T. J. Hellyer and J. G. Nadeau, Expert. Rev. Mol. Diagn., 2004, 4, 251-261.

16 V. V. Demidov, Expert. Rev. Mol. Diagn., 2005, 5, 477-478.
17 L. Bissonnette and M. G. Bergeron, Expert. Rev. Mol. Diagn., 2006, 6, 433-450.

18 K. K. Jain, Expert. Rev. Mol. Diagn., 2003, 3, 153-161.

19 R. Zhao, Clin. Lab. Sci., 2005, 18, 254-262.

20 M. L. Kopka, C. Yoon, D. Goodsell, P. Pjura and R. E. Dickerson, Proc. Natl. Acad. Sci. U. S. A., 1985, 82, 1376-1380.

21 M. Mrksich and P. B. Dervan, J. Am. Chem. Soc., 1993, 115, 9892-9899.

22 S. White, J. W. Szewczyk, J. M. Turner, E. E. Baird and P. B. Dervan, Nature, 1998, 391, 468-471.

23 S. Janssen, T. Durussel and U. K. Laemmli, Mol. Cell, 2000, 6, 999-1011.

24 K. Maeshima, S. Janssen and U. K. Laemmli, EMBO J., 2001, 20, $3218-3228$.

25 E. J. Fechter, B. Olenyuk and P. B. Dervan, J. Am. Chem. Soc., 2005, 127, 16685-16691.

26 V. C. Rucker, S. Foister, C. Melander and P. B. Dervan, J. Am. Chem. Soc., 2003, 125, 1195-1202.

27 C. L. Warren, N. C. Kratochvil, K. E. Hauschild, S. Foister, M. L. Brezinski, P. B. Dervan, G. N. Phillips, Jr. and A. Z. Ansari, Proc. Natl. Acad. Sci. U. S. A., 2006, 103, 867-872.

28 T. Le Doan, L. Perrouault, D. Praseuth, N. Habhoub, J. L. Decout, N. T. Thuong, J. Lhomme and C. Helene, Nucleic Acids Res., 1987, 15, 7749-7760.

29 H. E. Moser and P. B. Dervan, Science, 1987, 238, 645-650.

30 A. J. Cheng, J. C. Wang and M. W. Van Dyke, Antisense Nucleic Acid Drug Dev., 1998, 8, 215-225.

31 M. D. Johnson, Chromosoma, 1999, 108, 181-189.

32 C. Escude, T. Garestier and C. Helene, Proc. Natl. Acad. Sci. U. S. A., 1999, 96, 10603-10607.

33 B. Geron-Landre, T. Roulon, P. Desbiolles and C. Escude, Nucleic Acids Res., 2003, 31, e125.

34 B. Geron-Landre, T. Roulon and C. Escude, FEBS J., 2005, 272, $5343-5352$.

35 J. S. Li, F. X. Chen, R. Shikiya, L. A. Marky and B. Gold, J. Am. Chem. Soc., 2005, 127, 12657-12665.

36 T. Roulon, C. Helene and C. Escude, Angew. Chem., Int. Ed., 2001, 40, 1523-1526.

37 J. I. Daksis and G. H. Erikson, Genet. Test., 2005, 9, 111-120.

38 E. Cassuto, S. C. West, J. Podell and P. Howard-Flanders, Nucleic Acids Res., 1981, 9, 4201-4210.

39 B. J. Rao, S. K. Chiu and C. M. Radding, J. Mol. Biol., 1993, 229, 328-343.

40 P. E. Nielsen, M. Egholm, R. H. Berg and O. Buchardt, Science, 1991, 254, 1497-1500.

41 Y. Shigemori, H. Haruta, T. Okada and M. Oishi, Genome Res., 2004, 14, 2478-2485.

42 E. S. Baker, J. W. Hong, B. S. Gaylord, G. C. Bazan and M. T. Bowers, J. Am. Chem. Soc., 2006, 128, 8484-8492.

43 P. Schatz, J. Distler, K. Berlin and M. Schuster, Nucleic Acids Res., 2006, 34, e59.

44 E. Y. Chan, N. M. Goncalves, R. A. Haeusler, A. J. Hatch, J. W. Larson, A. M. Maletta, G. R. Yantz, E. D. Carstea, M. Fuchs, G. G. Wong, S. R. Gullans and R. Gilmanshin, Genome Res., 2004, 14, 1137-1146.

45 K. E. Lundin, L. Good, R. Stromberg, A. Graslund and C. I. Smith, Adv. Genet., 2006, 56, 1-51.

46 J. C. Venter, Science, 2001, 291, 1304-1351

47 M. Thompson, Bioconjugate Chem., 2006, 17, 507-513.

48 J. R. Taylor, M. M. Fang and S. Nie, Anal. Chem., 2000, 72, 1979-1986.

49 C. C. Robinett, A. Straight, G. Li, C. Willhelm, G. Sudlow, A. Murray and A. S. Belmont, J. Cell Biol., 1996, 135, 1685-1700.

50 A. S. Gonzalez-Serricchio and P. W. Sternberg, BMC Genet., 2006, 7, 36 .

51 A. T. Ooi, C. I. Stains, I. Ghosh and D. J. Segal, Biochemistry, 2006, 45, 3620-3625.

52 C. I. Stains, J. L. Furman, D. J. Segal and I. Ghosh, J. Am. Chem. Soc., 2006, 128, 9761-9765.

53 C. I. Stains, J. R. Porter, A. T. Ooi, D. J. Segal and I. Ghosh, J. Am. Chem. Soc., 2005, 127, 10782-10783.

54 S. A. Wolfe, L. Nekludova and C. O. Pabo, Annu. Rev. Biophys. Biomol. Struct., 2000, 29, 183-212.

55 R. R. Beerli and C. F. Barbas, Nat. Biotechnol., 2002, 20, 135-141. 
56 C. O. Pabo, E. Peisach and R. A. Grant, Annu. Rev. Biochem., 2001, 70, 313-340.

57 B. Dreier, R. R. Beerli, D. J. Segal, J. D. Flippin and C. F. Barbas III, J. Biol. Chem., 2001, 276, 29466-29478.

58 B. Dreier, R. P. Fuller, D. J. Segal, C. V. Lund, P. Blancafort, A. Huber, B. Koksch and C. F. Barbas, J. Biol. Chem., 2005, 280, 35588-35597.

59 D. J. Segal, B. Dreier, R. R. Beerli and C. F. Barbas III, Proc. Natl. Acad. Sci. U. S. A., 1999, 96, 2758-2763.

60 M. Mani, K. Kandavelou, F. J. Dy, S. Durai and S. Chandrasegaran, Biochem. Biophys. Res. Commun., 2005, 335, 447-457.

61 D. J. Segal, Methods, 2002, 26, 76-83.

62 I. Remy, A. Galarneau and S. W. Michnick, Methods Mol. Biol., 2002, 185, 447-459.

63 I. Ghosh, A. D. Hamilton and L. Regan, J. Am. Chem. Soc., 2000, 122, 5658-5659.

64 W. Gao, B. Xing, R. Y. Tsien and J. Rao, J. Am. Chem. Soc., 2003, 125, 11146-11147.

65 A. Galarneau, M. Primeau, L. E. Trudeau and S. W. Michnick, Nat. Biotechnol., 2002, 20, 619-622.

66 A. Bird, Cell, 1992, 70, 5-8.

67 A. P. Bird, Nucleic Acids Res., 1980, 8, 1499-1504.

68 R. Jaenisch and A. Bird, Nat. Genet., 2003, 33 Suppl, 245-254.

69 S. J. Clark, J. Harrison, C. L. Paul and M. Frommer, Nucleic Acids Res., 1994, 22, 2990-2997.
70 Y. Furuichi, Y. Wataya, H. Hayatsu and T. Ukita, Biochem. Biophys. Res. Commun., 1970, 41, 1185-1191.

71 J. G. Herman, J. R. Graff, S. Myohanen, B. D. Nelkin and S. B. Baylin, Proc. Natl. Acad. Sci. U. S. A., 1996, 93, 9821-9826.

72 Z. Xiong and P. W. Laird, Nucleic Acids Res., 1997, 25, 2532-2534.

73 A. M. Raizis, F. Schmitt and J. P. Jost, Anal. Biochem., 1995, 226, $161-166$.

74 H. Thomassin, E. J. Oakeley and T. Grange, Methods, 1999, 19, 465-475.

75 T. Rein, D. A. Natale, U. Gartner, M. Niggemann, M. L. DePamphilis and H. Zorbas, J. Biol. Chem., 1997, 272, 10021-10029.

76 M. F. Fraga and M. Esteller, Biotechniques, 2002, 33, 632, 634, 636-649.

77 P. M. Warnecke, C. Stirzaker, J. Song, C. Grunau, J. R. Melki and S. J. Clark, Methods, 2002, 27, 101-107.

78 H. J. Tanke, R. W. Dirks and T. Raap, Curr. Opin. Biotechnol., 2005, 16, 49-54.

79 T. G. Uil, H. J. Haisma and M. G. Rots, Nucleic Acids Res., 2003, 31, 6064-6078.

80 A. F. Kolb, C. J. Coates, J. M. Kaminski, J. B. Summers, A. D. Miller and D. J. Segal, Trends Biotechnol., 2005, 23, 399-406.

81 V. M. Vrudhula, D. E. Kerr, N. O. Siemers, G. M. Dubowchik and P. D. Senter, Bioorg. Med. Chem. Lett., 2003, 13, 539-542. 